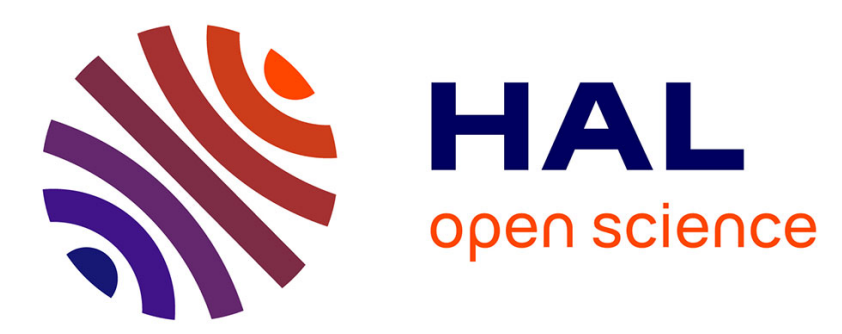

\title{
Metal-promoted coupling reactions implying ligand-based redox changes
}

Jérémy Jacquet, Marine Desage-El Murr, Louis Fensterbank

\section{To cite this version:}

Jérémy Jacquet, Marine Desage-El Murr, Louis Fensterbank. Metal-promoted coupling reactions implying ligand-based redox changes. ChemCatChem, 2016, 8 (21), pp.3310-3316 10.1002/cctc.201600616 . hal-01371934

\section{HAL Id: hal-01371934 https://hal.sorbonne-universite.fr/hal-01371934}

Submitted on 26 Sep 2016

HAL is a multi-disciplinary open access archive for the deposit and dissemination of scientific research documents, whether they are published or not. The documents may come from teaching and research institutions in France or abroad, or from public or private research centers.
L'archive ouverte pluridisciplinaire HAL, est destinée au dépôt et à la diffusion de documents scientifiques de niveau recherche, publiés ou non, émanant des établissements d'enseignement et de recherche français ou étrangers, des laboratoires publics ou privés. 


\title{
Metal-promoted coupling reactions implying ligand-based redox changes
}

\author{
Jérémy Jacquet, ${ }^{[\mathrm{a}]}$ Marine Desage-El Murr ${ }^{[\mathrm{a}] \star}$ and Louis Fensterbank ${ }^{[\mathrm{a}] \star}$
}

\begin{abstract}
Coupling reactions are staples in the synthetic world and their efficiency relies on well-defined, mostly bis-electronic, elementary catalytic steps. An area of great interest currently lies in the taming of radical species and their efficient introduction in catalytic cycles. Among these species bearing radical character, redox-active ligands hold much potential and can be used to sustain synthetically relevant couplings by introducing ligand-based electronic contribution. This minireview aims at presenting the current state of this promising field.
\end{abstract}

\section{Introduction}

Since their original discovery in the early eighties, transition metal catalyzed coupling reactions have achieved iconic status and forged their way to become classics in synthetic chemistry. The pinnacle of this area was reached with the attribution of the 2010 Nobel Prize in Chemistry for outstanding contribution of cross-coupling methodologies to the field of carbon-carbon (and other) bond forming events. Among the myriad of catalytic processes developed, carbon-carbon and carbon-heteroatom bond formation are almost textbook applications, which have garnered sustained attention over the years due to their deceptive simplicity. Establishing the best catalytic system for a given cross-coupling typically requires finding the best combination of metal precatalyst and ligand (or preformed catalyst), solvent and sometimes additives such as base. Among these, the role of the ligand is of paramount importance as its geometry -and related parameters such as bulkiness, hapticity, bite angle, buried volume and such- as well as its electronics (Lewis acidity or basicity, electron-rich or electron-deficient...) can greatly influence the outcome of the reaction. Regarding ligands, popular choices include phosphine and related phosphorous-based derivatives as well as $\mathrm{N}$-heterocyclic carbenes.

While the span of effective metals includes nearly every transition metal, noble metals and especially palladium have enjoyed a blossoming development, partly due to their unrivalled efficiency in bis-electronic elementary catalytic steps such as oxidative addition and reductive elimination. However, in the wake of the foreseen shortage of precious metals, the stringent need for alternative catalytic systems based on earth-abundant metals has led chemists to explore the possibility of expanding these metals' applicability and, among other strategies, through

[a] J. Jacquet, Dr M. Desage-El Murr, Prof. L. Fensterbank Sorbonne Universités, UPMC, Université Paris 06,UMR CNRS 8232, Institut Parisien de Chimie Moléculaire, France.

E-mail: marine.desage-el_murr@upmc.fr

louis.fensterbank@upmc.f the use of redox ligands. Akin to the redox cofactors encountered in Nature, these ligands can provide additional electronic density to the metal, thus circumventing the metal's tendency to perform single-electron transfer (SET). ${ }^{[1]}$

These so-called redox non-innocent ligands are enjoying a steady development. However, their application in broad scope synthetic endeavors such as cross-coupling is not streamlined yet and sometimes remains stoichiometric in metal complex. This is partly due to the difficulty of analyzing the resulting mechanism in great detail, as the paramagnetic intermediates involved can be very elusive and often require the use of advanced techniques such as EPR. Also, the added complexity generated by the redox ligand can complicate some elementary steps of the mechanism and hinder synthetic development. The detailed understanding of such systems should however lead to better catalytic systems and possibly avoid the pitfalls associated with optimization of the system when mechanistic insights have not provided a clear picture of the processes at stake.

This review focuses on intermolecular coupling events including cross- and homo-couplings in which the redox activity of the ligand is explicitly mentioned. Cycloisomerizations will not be covered and transformations in which the bond formation takes place on the ligand are beyond the scope of this review.

\section{2. $\mathrm{Csp}^{2}-\mathrm{Csp}^{2}$ coupling}

\subsection{Nickel}

Bielawski and co-workers have described the synthesis of a nickel complex bearing a $\mathrm{N}$-heterocyclic carbene ligand with a built-in redox-active naphtoquinone subunit. ${ }^{[2]}$ The resulting complex successfully performs aryl-aryl Kumada cross-coupling and strikingly, the catalytic activity can be switched on and off by external redox stimulus. A combination of UV-vis and electrochemical studies were performed on this "arrested catalysis", as referred to by the authors, and the mechanism (scheme 1) is proposed to proceed through a catalytic cycle in which a Ni-NHC intermediate $\mathbf{3}$-presumably arising from oxidative addition of the aryl halide on the starting Ni speciescan be reduced by addition of $\left[\mathrm{CoCp}_{2}\right]$ in the reaction medium, thus becoming catalytically inactive. However, subsequent reoxidation with $[\mathrm{Fc}]\left[\mathrm{BF}_{4}\right]$ reinstates the initial catalytic activity. The yields range from 67 to $98 \%$, although the scope has not been extensively evaluated. While the electronic contribution from the ligand does not participate to the product formation, this unusual example of control of catalytic properties through redox stimulus opens the way towards fruitful developments. 
Jérémy Jacquet was born in 1989, in Normandy, France. In 2013, he graduated from Chimie ParisTech (ENSCP) and received the same year his M.Sc degree in molecular chemistry from the Université Pierre et Marie Curie (UPMC, Paris VI). In October 2013, he started his Ph.D. research at the Institut Parisien de Chimie Moléculaire (IPCM, UPMC) under the supervision of Prof. Louis Fensterbank and Dr. Marine Desage-El Murr. His work focuses on organometallic catalysis using complexes bearing non-innocent amidophenolate ligands.

Marine Desage-El Murr studied at the Ecole Supérieure de Chimie Physique et Electronique de Lyon (CPE Lyon), and Ecole Nationale Supérieure de Chimie de Paris (ENSCP). She obtained her PhD in 2003 under the supervision of $\mathrm{Dr}$ Charles Mioskowski at the Commissariat à l'Energie Atomique (CEA Saclay). Since 2007, she is Assistant Professor at the Institut Parisien de Chimie Moléculaire (IPCM) at the Université Pierre et Marie Curie (UPMC). Her research interests encompass organometallic catalysis and catalytic systems and particularly redox ligands.

Louis Fensterbank obtained his Ph.D. in 1993 from SUNY Stony Brook with Scott Sieburth. After a temporary lecturer position at UPMC in 1994, he was appointed Chargé de Recherche CNRS in 1995 in Max Malacria's team. In 2004, he obtained a professorship position at UPMC and in 2008, he was nominated junior member of the Institut Universitaire de France. In 2014, he reeived the Clavel Lespiau Prize from the Académie des Sciences. He is the co-author of 190 publications
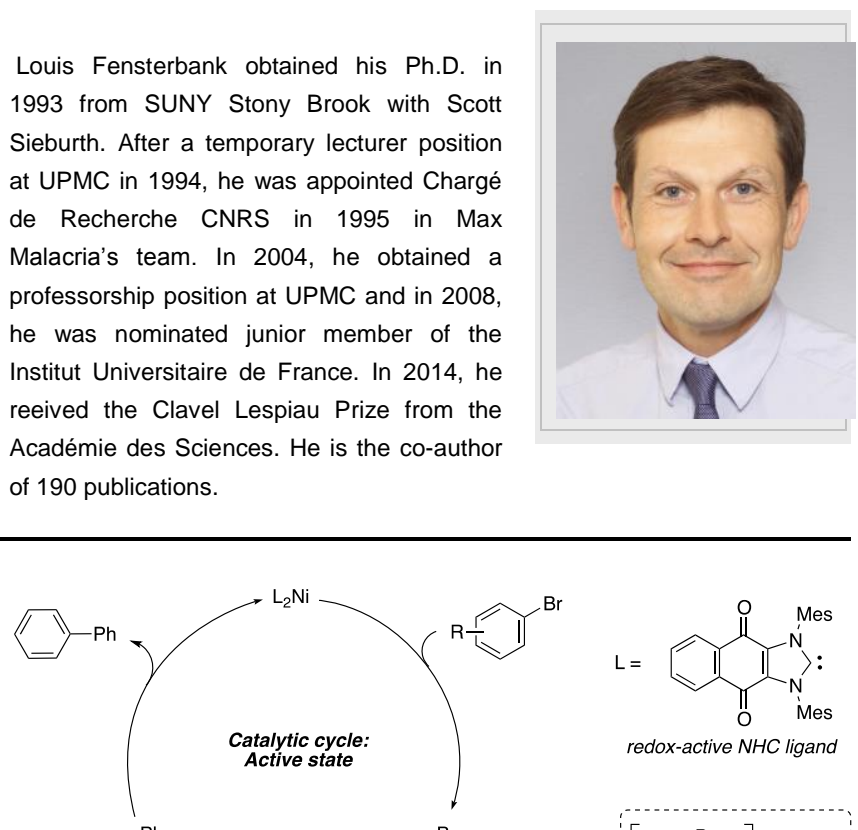

redox-active NHC ligand
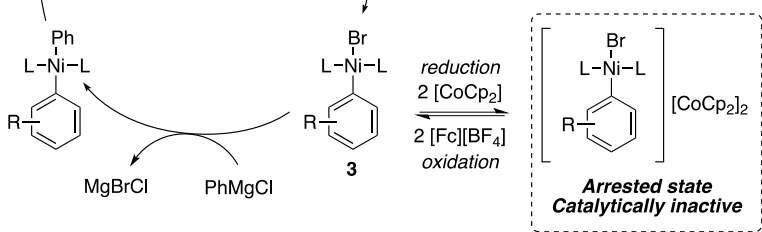

Scheme 1. Mechanism for the arrested catalysis in nickel-catalyzed Kumada cross-coupling with a redox-switchable ligand.
The non-innocent role of $\alpha$-diimine ligands and specifically bipyridine ligands (bpy) in organonickel complexes has been documented in the context of $\mathrm{C}-\mathrm{C}$ bond formation in the study of homocoupling of aryl halides (scheme 2) ${ }^{[3]}$ In depth analysis of the electrogenerated species by a combination of UV-vis, spectroelectrochemistry and EPR led the authors to conclude on a ligand-centered reduction event upon electrochemical reduction of a [Ni" $(\mathrm{Ar}) \mathrm{Br}(\mathrm{bpy})]$ species, similar to those implied in the catalytic cycle after the first step of oxidative addition of the aryl halide to the starting nickel (0) complex. Electroreduction of [Nill(Ar)Br(bpy)] leads to a species bearing significant radical character which the authors ascribe to a SOMO centered on the bpy $\left(\pi^{*}\right)$ of the diimine ligand. ${ }^{[4]}$

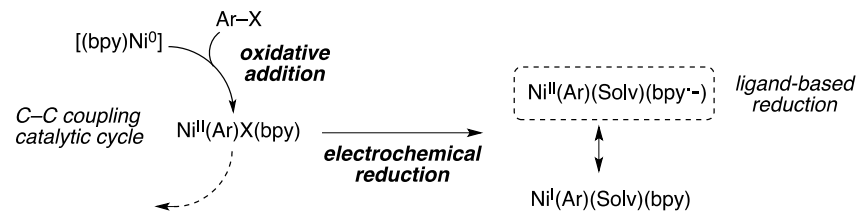

Scheme 2. Electrochemical reduction of nickel-based intermediates for mechanism elucidation.

\subsection{Palladium}

Gupta and co-workers have investigated the activity of palladium complexes with 13-membered amide-based macrocyclic ligands in Suzuki and Heck cross-couplings. ${ }^{[5]}$ A family of four complexes (4-7, scheme 3) differing by the electronic substituents on the aromatic bridging ring was prepared and preliminary investigations on their electronic behavior in solution were performed. Interestingly, only the ligand bearing strong electron-donating methoxy substituents on the ligand core (complex 7) was found to sustain ligand-based oxidation, evidenced by a sharp isotropic signal at $g=2.011$ observed in EPR spectroscopy. This is an interesting difference of behavior within a family of structurally close ligand scaffolds. All complexes were tested in cross-coupling and were found to have moderate activity, with good yields being obtained with aryl iodides as electrophiles. While modest, the catalytic activity of complex 7 shows that the redox interplay probably occurring between the ligand and palladium center is compatible with a catalytic activity and more fine-tuning of the ligand could probably be beneficial. Especially, mechanistic investigations suggest that no $\operatorname{Pd}(0)$ species occur in the reaction medium, which points towards a palladium redox couple distinct from the $\mathrm{Pd}^{\prime \prime} / \mathrm{Pd}^{0}$ classically involved in cross-couplings.
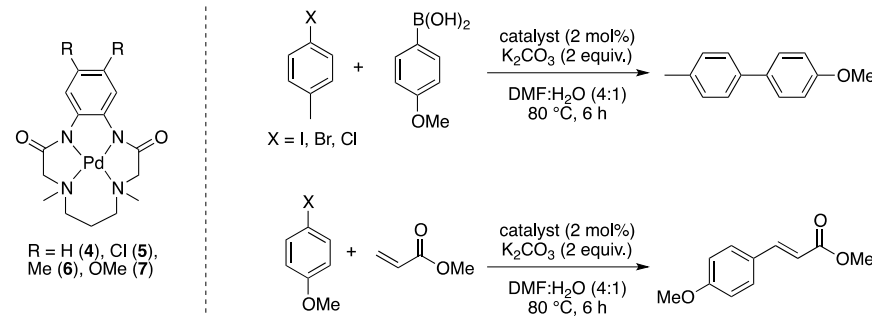

Scheme 3. Structure of palladium complexes with amide-based macrocyclic ligands and representative coupling reactions. 
A recent example of an unusual bimetallic complex of iridium and palladium was reported by Tejel, de Bruin and co-workers (Scheme 4). ${ }^{[6]}$ The authors had previously isolated the parent anionic $\mathrm{Ir}^{\prime}$ complex $\mathbf{1 0}$ with a dianionic ligand corresponding to the doubly reduced form of an iminopyridine ligand. ${ }^{[7,8]}$ Upon reaction of complex 10 with $\mathrm{PdCl}_{2}\left(\mathrm{PPh}_{3}\right)_{2}$, bimetallic complex 11 was isolated and its structure was elucidated by single crystal $X$ ray diffraction analysis. Quite counter intuitively, the metallic oxidation states within $\mathbf{1 1}$ were identified as $\mathrm{Ir}^{\mathrm{l}} / \mathrm{Pd}^{0}$, thus showing that the palladium center is reduced upon coordination to the iridium-bisiminopyridine fragment. The authors attribute this reduction to an internal two-electron transfer to the Pd" center, which then leads to a $\mathrm{Pd}^{0}$ center with a trigonal planar geometry. This type of palladium species bears resemblance to the tricoordinate anionic $\mathrm{Pd}^{0}$ species $\left[\mathrm{Pd}^{0} \mathrm{X}\left(\mathrm{PR}_{3}\right)_{3}\right]^{-}$which have been proposed to be intermediates in Heck-type palladium catalyzed coupling. Indeed, complex 11 was found to perform Heck reaction between phenyl iodide and an alkene, albeit in moderate yields.
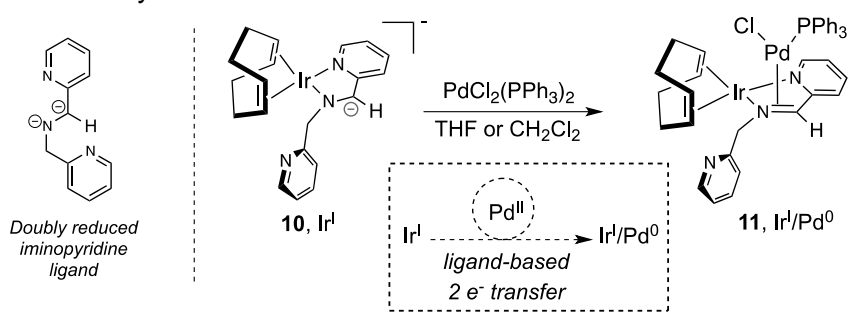

Scheme 4. Bimetallic complex 11 with $\operatorname{~Ir}^{1} / \mathrm{Pd}^{0}$ core used in Heck reactions.

The electronic effect of ferrocene groups introduced in macrocyclic triazatriolefinic structures was reported by Roglans and co-workers. ${ }^{[9]}$ Palladium complexes bearing ligands substituted by up to three ferrocene moieties were evaluated in Heck and Suzuki cross-couplings (Scheme 5). Subsequent cyclic voltammetry studies showed that the ferrocenyl groups had an influence on the redox properties of the palladium complexes.

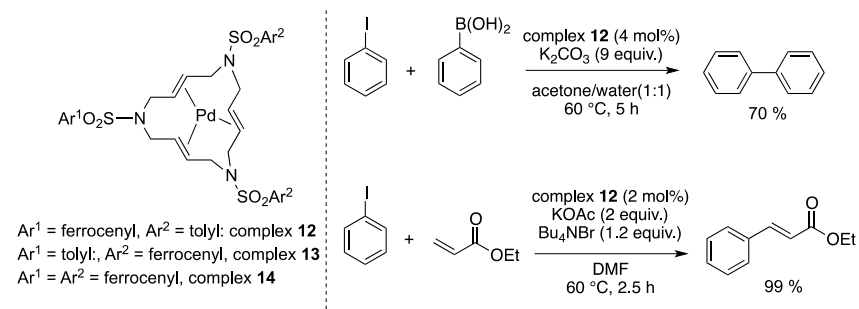

Scheme 5. Redox influence of ferrocenyl groups on palladium complexes for Suzuki-Miyaura and Heck cross-couplings.

\subsection{Group 4 metals (Ti, $\mathrm{Zr}, \mathrm{Hf})$}

Early contributions from the Heyduk group have established the ability of amidophenolate redox ligands to sustain a formal oxidative addition $^{[10]}$ and subsequent reductive elimination ${ }^{[11]}$ at a $d^{0} \mathrm{Zr}(\mathrm{IV})$ complex (Scheme 6).

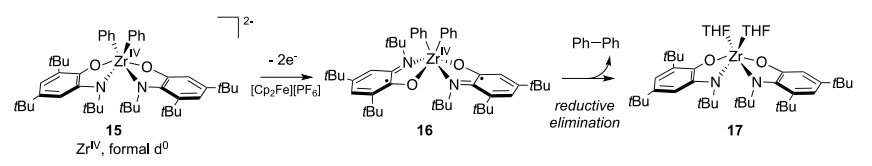

Scheme 6. Biphenyl reductive elimination occuring at a $\mathrm{Zr}^{\mathrm{IV}}$ center bearing iminosemiquinone ligands.

Ligand-centered oxidation of starting complex 15 containing amidophenolate ligands by ferrocenium results in a doubly oxidized complex 16 bearing two iminosemiquinone ligands. Triggered by the oxidation of the redox ligands, biphenyl formation occurs from this intermediate complex 16 and crossover experiments carried out with tolyl instead of phenyl substituents evidenced that coordination to the metal center was mandatory for the reaction to proceed, thus underlying the crucial role of the ligands. However, the related reductive elimination from analogs of complex 15 with methyl instead of phenyl groups did not occur, which the authors attribute to competing formation of methyl radicals, thus ruling out $\mathrm{Csp}^{3}-$ $\mathrm{Csp}^{3}$ coupling so far. Later computational work from Baik and co-workers on $\mathrm{d}^{4}$ metals ( $\mathrm{Ti}, \mathrm{Zr}$ and $\mathrm{Hf}$ ) bearing similar ligands (2,4-di-tert-butyl-6-tert-butyliminosemiquinone) further supported ligand-based redox events. ${ }^{[12]}$ The details of electronic transfer were studied and two possible mechanisms were considered: a pairwise electron-transfer and an unpaired electron-transfer. The pairwise electron-transfer was found to be favored but the authors observed that while not being directly involved in the redox processes, the metal had an influence on the reaction energetics.

\subsection{Iron}

Desage-El Murr, Fensterbank and co-workers described an efficient way to form $\mathrm{C}-\mathrm{C}$ bonds through a $\mathrm{C}-\mathrm{H}$ activation / $\mathrm{C}-\mathrm{C}$ coupling tandem reaction, catalyzed by iron complex 18 with a bisiminopyridine ligand (Scheme 7). ${ }^{[13]} \mathrm{Csp}^{2}-\mathrm{Csp}^{2}$ bond formation was achieved between benzene and aryl bromides, in the presence of KHMDS. Spectroscopic studies attested the formation of a paramagnetic species, likely resulting from an electron transfer from KHMDS to the starting complex, and radical probes experiments allowed the authors to exclude substrate-centered radicals. These results suggest an innersphere pathway for the $\mathrm{C}-\mathrm{H}$ activation step rather than a HAS (Homolytic Aromatic Substitution) reaction involving aryl radicals. This behavior, which is uncommon for first row transition metals, is also supported by DFT calculations and a three components transition state was proposed for the $\mathrm{C}-\mathrm{H}$ activation step: electron transfer from HMDS anion to the ligand of [LFe" $\left.^{-1} \mathrm{Br}\right]^{+}$ complex in a quintet state, leads to an antiferromagnetic coupling between the electron on the ligand and the HMDS radical. An hydrogen atom abstraction then occurs simultaneously with formation of a Fe-C bond. 


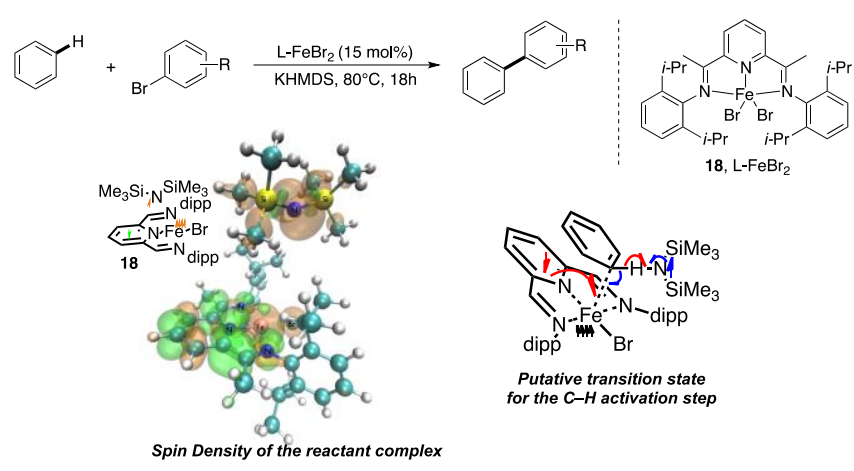

Scheme 7. $\mathrm{Csp}^{2}-\mathrm{Csp}^{2}$ bond formation by tandem $\mathrm{C}-\mathrm{H}$ activation/arylation with iron bisiminopyridine complex 18 and proposed transition state.

\section{3. $\mathrm{Csp}^{2}-\mathrm{Csp}^{3}$ coupling}

\subsection{Cobalt}

In 2010, the Soper group reported a Negishi-like cross-coupling, using a cobalt (III) complex $\mathbf{1 9}$ bearing two redox-active amidophenolate ligands (Scheme 8). ${ }^{[14]}$ The square planar complex, exhibiting a nucleophilic behavior, is able to react with alkyl halides under mild conditions to form a metal-alkyl bond. The two redox electrons furnished by the ligands during this oxidative addition step allow the metal center to preserve its original oxidation state. The complex characterized by ESI-MS and X-ray analysis is relatively stable and does not undergo $\beta$ elimination. However, C-C coupling was achieved when an organozinc reagent was added to a solution of the alkyl-cobalt (III) complex. The ligand-centered reduction restores the initial cobalt (III) complex along with the delivery of the coupling product. Experimental observations suggest a $\mathrm{S}_{\mathrm{N}} 2$-type mechanism for the oxidative step, but only allow to exclude radical homolysis of the alkyl-cobalt bond during the reductive step.

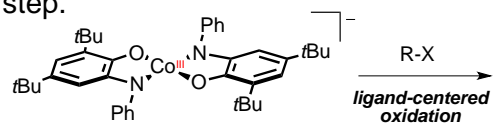
$19\left[\mathrm{Co}^{\prime \prime \prime}\left(\mathrm{L}_{\mathrm{AP}}\right)_{2}\right]^{\cdot}$

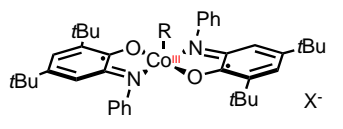

$$
20\left[\mathrm{Co}^{\prime \prime \prime}\left(\mathrm{L}_{\mathrm{SQ}}\right)_{2} \mathrm{R}\right]
$$

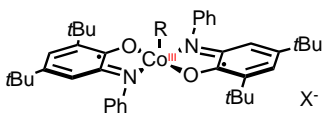

$20\left[\mathrm{Co}^{\prime \prime \prime}\left(\mathrm{L}_{\mathrm{SQ}}\right)_{2} \mathrm{R}\right]$
Scheme 8. Formal Negishi cross-coupling occuring at $\mathrm{Co}^{\prime \prime \prime}$ complex with aminophenolate ligands.

The reactivity of an aryl-cobalt complex 23 bearing a bisiminopyridine ligand has been investigated in cross-coupling by Budzelaar and co-workers (Scheme 9). ${ }^{[15]}$ Aryl cobalt complex 23 is easily accessible by reaction of the neutral species $\left(\mathrm{L}^{-}\right) \mathrm{Co}^{\prime}\left(\mathrm{N}_{2}\right) 22$ with an aryl chloride $\mathrm{ArCl}$, via a chloride abstraction step, followed by the capture of the free aryl radical by another neutral cobalt complex 22 leading to a mixture of $\left(\mathrm{L}^{-}\right.$ $\cdot) \mathrm{Co}$ "Ar 23 and $\left(\mathrm{L}^{-}\right) \mathrm{Co} " \mathrm{Cl} 24$. Complex 23 was shown to be reactive towards activated benzyl or allyl chlorides and bromides, providing mixtures of homo- and heterocoupling products. Interestingly, one example of $\mathrm{Csp}^{3}-\mathrm{Csp}^{3}$ was also achieved with $\left(\mathrm{L}^{-}\right) \mathrm{CoCH}_{2} \mathrm{SiMe}_{3}$ and $\mathrm{CH}_{3}$ l. The mechanism suggested by the authors starts with a halide abstraction, generating the unstable $(\mathrm{L}) \mathrm{Co}^{\prime \prime}(\mathrm{Ar})(\mathrm{Br})$ and a benzyl radical. Loss of an aryl radical, favored by the oxidant character of $L^{0}$, allows the recombination with the previous benzyl radical to form the $\mathrm{Csp}^{2}-\mathrm{Csp}^{3}$ bond.
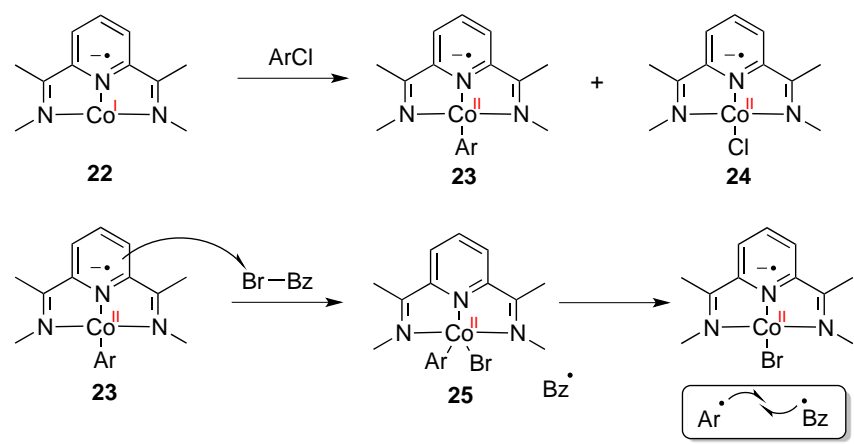

Scheme 9. $\mathrm{Csp}^{2}-\mathrm{Csp}^{3}$ coupling through radical recombination mediated by cobalt complex with a bisiminopyridine ligand.

\subsection{Nickel}

In 2012, Enthaler and co-workers studied the $\mathrm{Csp}^{2}-\mathrm{Csp}^{3}$ bond formation catalyzed by new nickel (II) complexes bearing 5hydroxypyrazine ligands (Scheme 10). ${ }^{[16]}$ The tridentate O,N,O' coordination mode with nickel results from a ring opening of the pyrazine core initiated by ammonia or 4-dimethylaminopyridine (DMAP) deprotonation. The base, acting also as a co-ligand, led to a diamagnetic square planar complex with ammonia, whereas a paramagnetic octahedral paramagnetic was obtained with DMAP.

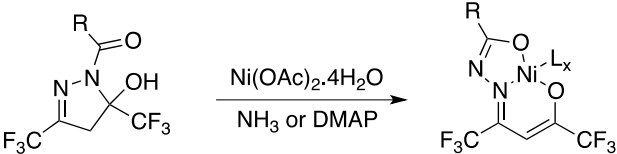

$$
\begin{aligned}
& \mathrm{R}=\mathrm{C}_{6} \mathrm{H}_{5}, \mathrm{CH}_{3} \\
& \mathrm{~L}=\mathrm{NH}_{3}(\mathrm{x}=1), \operatorname{DMAP}(\mathrm{x}=3)
\end{aligned}
$$

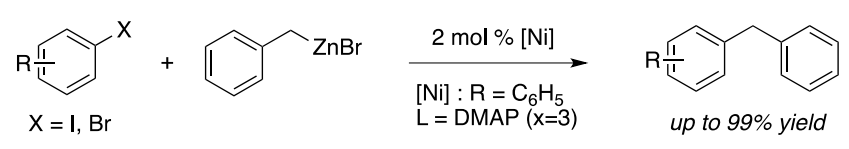

Scheme 10. Nickel-catalyzed $\mathrm{Csp}^{2}-\mathrm{Csp}^{3}$ bond-formation

These complexes were evaluated in $\mathrm{Csp}^{2}-\mathrm{Csp}^{3}$ cross-coupling catalysis with aryl halides and benzylzinc bromides or dialkylzinc reagents. Performing the reaction at $70^{\circ} \mathrm{C}$ with low catalytic loading (2 mol\%), good to excellent yields were obtained with aryl iodides and bromides. However, the reaction proved to be 
extremely slow with aryl chlorides and impossible with fluorides. DFT calculations, consistent with $\mathrm{X}$-ray structures, described degenerated SOMOs, with a main contribution from the nickel $d$ orbitals and some from the ligand for the lowest energy SOMO.

\section{4. $C s p^{3}-C_{s p}^{3}$ coupling}

\subsection{Nickel}

Nickel catalysts are well-known in cross-coupling. ${ }^{[17]}$ Exploiting their well-established ability to avoid unproductive $\beta-\mathrm{H}$ elimination pathways in cross-coupling reactions, the Vicic group reported a Negishi like $\mathrm{Csp}^{3}-\mathrm{Csp}^{3}$ bond-forming reaction catalyzed by nickel complexes with terpyridine ligands (Scheme 11). ${ }^{[18,19]}$ Later findings reported that ligand modification by introduction of tert-butyl groups on the terpyridine scaffold (tpy' ligand) allowed isolation of two species $\left[\left(\mathrm{tpy}^{\prime}\right) \mathrm{NiCH}_{3}\right]^{+}, \mathrm{I}^{-}$and [(tpy') $\mathrm{NiCH}_{3}$ ] and their reactivity towards alkylzinc reagents could thus be probed. ${ }^{[20]}$ The results showed that a two electronsbased mechanism was not operative thus ruling out an oxidative addition-reductive elimination sequence. Further mechanistic insights were obtained which allowed to assert that the formation of (tpy) $\mathrm{NiCH}_{3}$ occurs through a non-radical pathway by comproportionation of (tpy) $\mathrm{Ni}\left(\mathrm{CH}_{3}\right)_{2}$ and a $\mathrm{Ni}^{0}$ species. ${ }^{[21]}$ Also, extensive EPR and DFT studies unambiguously showed that this (tpy) $\mathrm{Ni}^{\prime} \mathrm{CH}_{3}$ species bears significant spin density on the tpy ligand and is best described as (tpy $\left.{ }^{-}\right) \mathrm{Ni}^{\prime \prime} \mathrm{CH}_{3}$, a nickel methyl cation coupled to a radical anion terpyridine. Based on these findings, the authors proposed a possible mechanistic scenario in which the key (tpy") $\mathrm{Ni}^{\prime \prime}$ (alkyl) species 26 performs the reduction of the alkyl halide, thus generating intermediate $\mathbf{2 7}$ together with an alkyl radical which then attacks the nickel center to generate the (tpy) $\mathrm{Ni}^{\prime \prime}(\mathrm{R})\left(\mathrm{R}^{\prime}\right) \mathbf{2 8}$ species poised for the final reductive elimination to yield the coupling product. The authors emphasize that the key feature of this coupling lies in the fact that the reduction of the alkyl halide is a ligand-based process and speculate that this specificity could translate into new forays in the cross-coupling synthetic field.
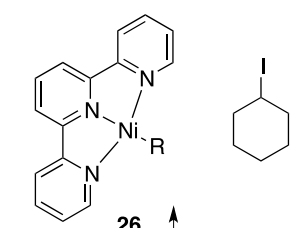

26
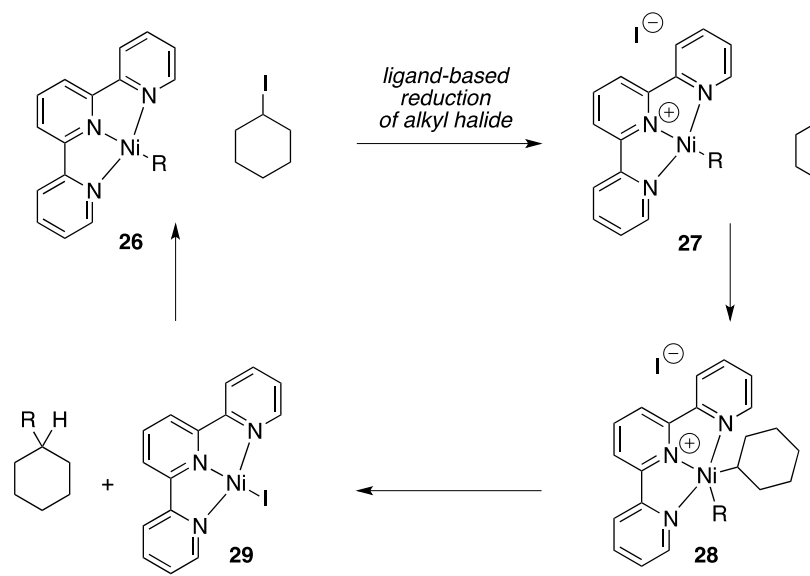

Scheme 11. Proposed mechanism involving ligand participation in nickelcatalyzed Negishi cross-coupling.

\subsection{Cobalt}

Generation of a redox-active cobalt (electro)catalyst has been described by the Sarkar group, and has been applied to a Csp ${ }^{3}$ $\mathrm{Csp}^{3}$ homocoupling reaction (Scheme 12). ${ }^{[22]}$ Electrochemical studies in $\mathrm{CH}_{2} \mathrm{Cl}_{2}$ showed that the cobalt (II) complex 30 , with its two diiminosemiquinone ligands, was able to support a first reversible reduction wave at $-1,47 \mathrm{~V} v s$. $\mathrm{Fc}^{+} / \mathrm{Fc}$ while a catalytic current was observed for the second reduction wave. This phenomenon was attributed to the catalytic reaction of the reduced complex with the solvent. Whereas the two reduction waves were found to be reversible in THF, the electrocatalysis was also noticed when benzyl bromide or chloride was added to a solution of complex $\mathbf{3 0}$ in THF. Kinetic studies allowed the authors to conclude to a first order reaction in halide concentration and the scan rate dependence of the observed rate constant suggested that the electrocatalyst was active only after the second reduction. The proposed mechanism starts with the ligand-centered reduction of $\left[\left(Q^{*}\right) C^{\prime \prime}\left(Q^{2}\right)\right]^{-} 31$ into the active complex $\left[\mathrm{Co}^{\prime \prime}\left(\mathrm{Q}^{2}\right)_{2}\right]^{2-} \mathbf{3 2}$. Then, reaction with benzyl halide via a ligand-centered oxidation, leads to the benzyl-cobalt complex $\left[\mathrm{Co}^{\prime \prime}\left(\mathrm{Q}^{*}\right)_{2} \mathrm{Bn}\right]^{-}$33, as confirmed by ESI mass spectroscopy. Finally, formation of the dibenzyl homocoupling product regenerates initial $\left[\left(Q^{*}\right) \mathrm{Co}^{\prime \prime}\left(\mathrm{Q}^{2}\right)\right]^{-}$complex 31 .

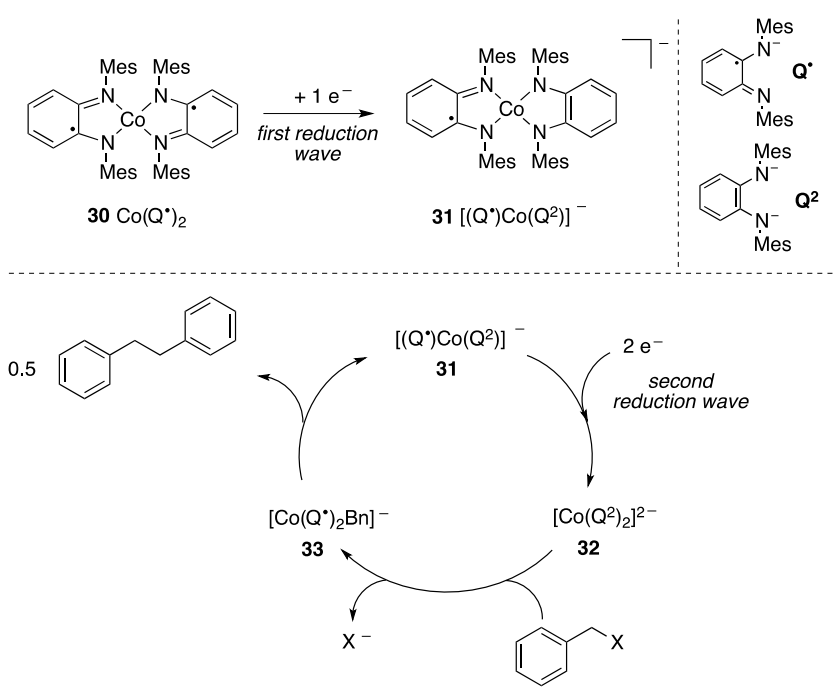

Scheme 12. Electrocatalytic generation of active cobalt complex for $\mathrm{Csp}^{3}$-Csp ${ }^{3}$ homocoupling.

\section{Conclusion}

Coupling is a typical organometallic catalytic application which enjoys many precedents in the literature. One of the current trends in the cross-coupling field, focusing on the development of catalytic systems based on earth-abundant metals, has fostered an interest in innovative (catalytic) manifolds relying on ligand-based electronic participation. As illustrated above, several families of ligands can be used for these transformations and provide promising results. Because the field of redox ligands 
is only lately transitioning from the stoichiometric to the catalytic field, not so many examples of cross-coupling reactions have been reported in which the redox role of the ligand is clearly established. A possible downside of this strategy is that electronic non-innocence can turn into chemical non-innocence, in this case leading to unwanted side reactions and/or sidetracking the reactivity of the catalyst. ${ }^{[23]}$

Among the advantages of this approach is the possibility to use redox-ligands as electron reservoirs in order to promote otherwise difficult or impossible reactivities, such as oxidative addition at a $\mathrm{d}^{0}$ metal center. ${ }^{[10]}$ This electron reservoir strategy may at first glance seem relevant mostly for first-row transition metals, which have a marked tendency towards single-electron transfer and therefore need an additional electron in order to engage in bis-electronic elementary steps such as oxidative addition or reductive elimination. However, noble metals are not without limitations. Current drawbacks associated with palladium-catalyzed cross-couplings are mostly due to its tendency to engage in $\beta-\mathrm{H}$ elimination pathways, which impairs $\mathrm{Csp}^{3}-\mathrm{Csp}^{3}$ cross-coupling. Redox ligands used in conjunction with palladium have triggered an unusual radical reactivity ${ }^{[24]}$ and could hold potential in future catalytic developments.

In conclusion, the use of redox ligands could have strong impact in the field of coupling reactions. Among the main challenges associated with this area is the combination of good catalytic activity coupled with in depth understanding of the electronics at stake in the reaction. However, this fast-developing field should witness significant progress and lead to a more streamlined approach towards building of molecular complexity.

\section{Acknowledgements}

The authors wish to thank CNRS, UPMC and MENRT (JJ).

Keywords: redox ligand $•$ cross-coupling $\cdot$ non-innocent ligand $•$ $\mathrm{C}-\mathrm{C}$ bond forming catalysis

[1] a) V. Lyaskovskyy, B. de Bruin, ACS Catal., 2012, 2, 270-279; b) P. J. Chirik, K. Wieghardt, Science, 2010, 327, 794-795; c) O. R. Luca, R. H. Crabtree, Chem. Soc. Rev., 2012, 42, 1440-1459.

[2] A. G. Tennyson, V. M. Lynch, C. W. Bielawski, J. Am. Chem. Soc. 2010 132, 9420-9429.

[3] A. Klein, Y. H. Budnikova, O. G. Sinyashin, J. Organomet. Chem. 2007, 692, 3156-3166.

[4] A. Klein, A. Kaiser, B. Sarkar, M. Wanner, J. Fiedler, Eur. J. Inorg. Chem. 2007, 2007, 965-976.

[5] S. Kumar, R. R. Jha, S. Yadav, R. Gupta, New J. Chem. 2015, 39, 2042-2051.

[6] C. Tejel, L. Asensio, M. P. del Río, B. de Bruin, J. A. López, M. A. Ciriano, Angew. Chem. Int. Ed. 2011, 50, 8839-8843.

[7] C. Tejel, M. P. del Río, M. A. Ciriano, E. J. Reijerse, F. Hartl, S. Záliš, D. G. H. Hetterscheid, N. Tsichlis i Spithas, B. de Bruin, Chem. - Eur. J. 2009, 15, 11878-11889.

[8] C. Tejel, M. A. Ciriano, M. P. del Río, D. G. H. Hetterscheid, N. Tsichlis i Spithas, J. M. M. Smits, B. de Bruin, Chem. - Eur. J. 2008, 14 10932-10936.

[9] A. Llobet, E. Masllorens, M. Moreno-Mañas, A. Pla-Quintana, M. Rodríguez, A. Roglans, Tetrahedron Lett. 2002, 43, 1425-1428.
[10] K. J. Blackmore, J. W. Ziller, A. F. Heyduk, Inorg. Chem. 2005, 44, 5559-5561

[11] M. R. Haneline, A. F. Heyduk, J. Am. Chem. Soc. 2006, 128, 84108411.

[12] D. C. Ashley, M.-H. Baik, Chem. - Eur. J. 2015, 21, 4308-4314.

[13] E. Salanouve, G. Bouzemame, S. Blanchard, E. Derat, M. Desage-EI Murr, L. Fensterbank, Chem. - Eur. J. 2014, 20, 4754-4761.

[14] a) A. L. Smith, K. I. Hardcastle, J. D. Soper, J. Am. Chem. Soc. 2010, 132, 14358-14360; For a highlight, see: b) W. I. Dzik, J. I. van der Vlugt, J. N. H. Reek, B. de Bruin, Angew. Chem., Int. Ed. 2011, 50, 33563358.

[15] D. Zhu, I. Thapa, I. Korobkov, S. Gambarotta, P. H. M. Budzelaar, Inorg. Chem. 2011, 50, 9879-9887.

[16] C. I. Someya, S. Inoue, S. Krackl, E. Irran, S. Enthaler, Eur. J. Inorg. Chem. 2012, 1269-1277.

[17] X. Hu, Chem. Sci. 2011, 2, 1867-1886.

[18] T. J. Anderson, G. D. Jones, D. A. Vicic, J. Am. Chem. Soc. 2004, 126, 8100-8101.

[19] T. J. Anderson, G. D. Jones, D. A. Vicic, J. Am. Chem. Soc. 2004, 126, 11113-11113.

[20] G. D. Jones, C. McFarland, T. J. Anderson, D. A. Vicic, Chem. Commun. 2005, 4211-4213.

[21] G. D. Jones, J. L. Martin, C. McFarland, O. R. Allen, R. E. Hall, A. D. Haley, R. J. Brandon, T. Konovalova, P. J. Desrochers, P. Pulay, D. A. Vicic, J. Am. Chem. Soc. 2006, 128, 13175-13183.

[22] M. van der Meer, Y. Rechkemmer, I. Peremykin, S. Hohloch, J. van Slageren, B. Sarkar, Chem. Commun. 2014, 50, 11104-11106.

[23] a) T. W. Myers, G. M. Yee, L. A. Berben, Eur. J. Inorg. Chem. 2013 3831-3835; b) P. Chaudhuri, E. Bill, R. Wagner, U. Pieper, B. Biswas, T. Weyhermüller, Inorg. Chem. 2008, 47, 5549-5551.

[24] a) D. L. J. Broere, B.de Bruin, J. N. H. Reek, M. Lutz, S. Dechert, J. I. van der Vlugt, J. Am. Chem. Soc. 2014, 136, 11574-11577; b) D. L. J. Broere, S. Demeshko, B.de Bruin, E. A. Pidko, J. N. H. Reek, M. A Siegler, M. Lutz, J. I. van der Vlugt, Chem. Eur. J. 2015, 21, 58795886; c) D. L. J. Broere, L. L.Metz, B. de Bruin, , J. N. H. Reek, M. A. Siegler, J. I. van der Vlugt, Angew. Chem. Int. Ed. 2014, 54, 1516-1520. 